\title{
Blood Cholesterol Levels of Family Members of Stroke Patients in Suburban Areas
}

Krongkaew Thongruangsooksai, M.D., Wantem Sangkhao, R.N., Mayured Piromthong, R.N.

Division of Social Medicine, Trang Hospital, Mueang, Trang 92000, Thailand.

Received 14 March 2020 • Revised 6 July 2020 • Accepted 8 August 2020 • Published online 29 January 2021

\begin{abstract}
:
Objective: (1) To study dyslipidemia prevalence of members in stroke patients' families (2) To investigate factors related to dyslipidemia of members in stroke patients' families

Material and Methods: In this cross-sectional analytical study, after patients suffered from a stroke, the subjects were visited, interviewed through a questionnaire, and blood was drawn for testing, without fasting. This was in order to establish their cholesterol, high-density lipoprotein cholesterol (HDL-C), low-density lipoprotein cholesterol (LDL-C), and random plasma glucose. This also included a physical checkup and risk assessment for cardiovascular disease. The data were collected from 120 subjects.

Results: Prevalence of high total cholesterol level was $34.2 \%$, low HDL-C was $15.8 \%$, high LDL-C level was $37.5 \%$, and prevalence of dyslipidemia was $51.7 \%$. The samples were 76 blood relatives and 44 non-blood relatives in the patients' families. The average LDL-C level was $147.12 \pm 39.06$ and $31.7 \%$ of them suffered from Class 1 obesity, and $15.8 \%$ of them faced Class 2 obesity. According to Thai CV risk scores, the results revealed that $80.8 \%$ of the samples had a low risk, at $<10.0 \%$. The factors related to dyslipidemia were underlying diseases, and most groups of underlying diseases were hypertension and diabetes mellitus, respectively.
\end{abstract}

Conclusion: Prevalence of dyslipidemia in family Members of Stroke Patients was $51.7 \%$. Detection of this abnormality is important to them. This would help in designing a prevention plan, as well as to raise awareness of stroke prevention in family members of stroke patients.

Keywords: cholesterol level, dyslipidemia, family members of stroke

Contact: Krongkaew Thongruangsooksai, M.D.

Division of Social Medicine, Trang Hospital, Mueang, Trang 92000, Thailand.

E-mail: jkkjeab@gmail.com

() 2020 JHSMR. Hosting by Prince of Songkla University. All rights reserved.

This is an open access article under the CC BY-NC-ND license

(http://www.jhsmr.org/index.php/jhsmr/about/editorialPolicies\#openAccessPolicy).
J Health Sci Med Res 2021;39(3):229-243 doi: 10.31584 /jhsmr.2021782 www.jhsmr.org 


\section{Introduction}

Dyslipidemia is a risk factor for atherosclerosis, and leads to cerebrovascular diseases; such as stroke. ${ }^{1,2}$ Strokes are now a serious, global public health problem, and the World Stroke Organization has reported that strokes are the second leading cause of death in the world. ${ }^{3}$ In Thailand, strokes are also a major public health issue, and require a lot of resources for medical treatments. ${ }^{4}$ It could be seen that one of the risk factors affecting stroke is dyslipidemia. ${ }^{5-10}$ According to the experts of the Adult Treatment Panel as well as the National Cholesterol Education program in the United States, it has been suggested that people aged over 20 should have blood testing to check their lipid profiles; as most people with dyslipidemia have invisible symptoms. ${ }^{11}$ Prevalence of dyslipidemia can be found in every age group including those of a young age group. In fact, a screening test for dyslipidemia should normally be considered for every age group. ${ }^{12-19}$ The causes of dyslipidemia can be attributed to both genetic conditions and food consumption behavior of families. ${ }^{20-24}$ Therefore, it is of interest to view the lipid profiles of other family members of stroke patient, as it may outline factors related to dyslipidemia in said people. This would help in designing a prevention plan, so as to decrease stroke incidence, as well as to raise awareness of stroke prevention in said family members in the future.

\section{Material and Methods}

This was an analytical cross-sectional study.

The samples were selected by purposive sampling. The population in this study was family members of stoke patients, living outside of Trang Municipality which lies 10 to 40 kilometer from central Trang, Mueang district and, Trang province; from 1 October 2018-31 December 2019. The selection of the samples were calculated by the following formula

$$
\begin{aligned}
& n=\frac{Z^{2} P(1-P)}{d^{2}} \\
& n=\frac{1.96^{2} \times 0.5 \times(1-0.5)}{(0.1)^{2}} \approx 97 \text { samples }
\end{aligned}
$$

n: Sample size

$\mathrm{d}$ : Precision for prevalence

P: Expected prevalence

Z: Z statistic for a level of confidence

In this study: $Z=1.96, P=0.5$, and $d=0.1$

The inclusion criteria were as follows:

1. A family member living at the same house as a suffering patient. He orshe either genetically-related or a non-blood member, and having been living in the same house as a patient for at least three months.

2. $\mathrm{He} / \mathrm{she}$ was 20 years old or over.

3. He/she had normal perception, and could normally communicate with other people.

4. $\mathrm{He} /$ she was willing to participate in the study.

5. He/she was willing to have a blood test for a lipid profile and blood sugar.

The exclusion criteria were as follows:

1. A family member took cholesterol-lowering medication.

2. $\mathrm{He} /$ she had some following underlying diseases or conditions: biliary obstruction, nephrotic syndrome, hypothyroidism, hepatitis, and pregnancy.

3. He/she took some following medication: diuretic, cyclosporine, glucocorticoid, and amiodarone.

4. He/she had diabetes mellitus (DM) with poorly controlled disease.

5. He/she had hypertension (HT) with poorly controlled disease.

The samples for this study were family members of stoke patients; living outside of Trang Municipality, 
Mueang District and, Trang Province, including 15 subdistricts; from 1 October 2018-31 December 2019. Samples were selected by the aforementioned criteria. After patients have suffered from stroke, the samples (members of each family) were visited, interviewed through a questionnaire, and drawn for direct method of blood testing, without fasting. This was to establish the following: cholesterol, high-density lipoprotein cholesterol (HDL-C), low-density lipoprotein cholesterol (LDL-C) and random plasma glucose. We also conducted a physical checkup, and risk assessment of cardiovascular disease.

\section{Research instrument}

The questionnaire was divided into two parts:

Part 1: Interview of each sample.

Part 2: Blood testing results of each sample received from the International Organization for Standardization 15189-certified laboratory at Trang Hospital. The test for a serum level of total cholesterol, HDL-C, and LDL-C was conducted by the Direct Enzymatic Colorimetric method. ${ }^{25,26}$

\section{Measurements and definitions}

1. Blood relatives are the samples whom had lived in the same house as the stroke patients, and who were $1^{\text {st }}$ degree and $2^{\text {nd }}$ degree relations with the stroke patients.

2. Non-blood relatives are the samples who lived in the same house as the stroke patients, but were not related by blood.

3. Diagnostic level of hyperlipidemia were classified based on the guidelines of the Adult Treatment Panel III (ATP III) of the National Cholesterol Education Program (NCEP III). ${ }^{11}$ The cutoff points for parameters were defined as follows:

Total cholesterol level (TC) <200 mg/dL: desirable

TC 200-239 mg/dL: borderline high

$\mathrm{TC} \geq 240 \mathrm{mg} / \mathrm{dL}$ : high

HDL-C level $<40 \mathrm{mg} / \mathrm{dL}$ : low
LDL-C level <100 mg/dL: optimal

LDL-C level $\geq 160 \mathrm{mg} / \mathrm{dL}$ : high

Dyslipidemia is a disorder level of at least one type of cholesterol: total cholesterol level $\geq 240 \mathrm{mg} / \mathrm{dL}$, HDL-C level $<40 \mathrm{mg} / \mathrm{dL}$, LDL-C level $\geq 160 \mathrm{mg} / \mathrm{dL}$.

4. DM, with poorly controlled disease, was defined as: having a previous diagnosis of diabetes by a physician, and having a Hemoglobin $\mathrm{A} 1 \mathrm{C}(\mathrm{HbA} 1 \mathrm{C})$ level $>7.0 \%$ in the last three months, or without $\mathrm{HbA} 1 \mathrm{C}$ testing.

5. HT with poorly controlled disease was defined as: the latest blood pressure of systolic blood pressure $\geq 140$ millimeter of mercury $(\mathrm{mmHg})$, or diastolic blood pressure $\geq 90 \mathrm{mmHg}$

6. Weight, including clothing was measured by electronic balance weights with 0.1 kilogram accuracy.

7. Standing height, without shoes, to the nearest 0.1 centimeter $(\mathrm{cm})$ was measured by a wall-mounted height measure.

8. Waist circumference, taken around the body across the belly button; for which the criteria suitable for Asian people are $90 \mathrm{~cm}$ of male waist circumference, and $80 \mathrm{~cm}$ for females. ${ }^{27}$

9. Measurement of blood pressure is recommended for patients after have stopped drinking tea or coffee and smoking for 30 minutes, and have been at rest for 5 minutes. In addition, patients were required not to talk before or during the measurement. According to the methods of measurement, they placed an arm on the provided table, and an arm cuff was then used to record their heart rate. Blood pressure was measured by a mercury sphygmomanometer. Each patient's blood was pressure measured twice, with a 1-minute break in between. In case the readings of the $1^{\text {st }}$ and $2^{\text {nd }}$ systolic blood pressure were different by $5 \mathrm{mmHg}$ or over, a $3^{\text {rd }}$ measurement should be done, then the average of all readings were summed up. ${ }^{28}$ According to hypertension diagnosis by The Seventh Report of the Joint National Committee on Prevention, 
Detection, Evaluation, and Treatment of High Blood Pressure, hypertension is defined as systolic blood pressure $\geq 140$ $\mathrm{mmHg}$ and/or diastolic blood pressure $\geq 90 \mathrm{mmHg}{ }^{27,28}$

10. Body mass index (BMI) is calculated by weight in kilograms, divided by height in meters squared. Rates of $\mathrm{BMl}$ are as follows ${ }^{28}$ :

BMI $<18.5$ kilogram per meter square $\left(\mathrm{kg} / \mathrm{m}^{2}\right)$ means underweight.

$\mathrm{BMI}=18.5-22.90 \mathrm{~kg} / \mathrm{m}^{2}$ means a healthy weight.

$\mathrm{BMI}=23-24.90 \mathrm{~kg} / \mathrm{m}^{2}$ means overweight.

$\mathrm{BMI}=25-29.90 \mathrm{~kg} / \mathrm{m}^{2}$ means Class 1 obesity.

BMI $\geq 30 \mathrm{~kg} / \mathrm{m}^{2}$ means Class 2 obesity.

11. The 10-year Thai Cardiovascular (CV) Risk Score indicates risk factors of cardiovascular disease as future risks for Thai people. The results can estimate risks of illness, or death caused by coronary artery disease and stroke in a ten-year time frame. This score was created due to the study of risk factors affecting coronary artery disease, and stroke in Thai people under the project of the Electricity Generating Authority of Thailand Study conducted over 20 years; including looking for five risk levels of myocardial infarction, and fatal and non-fatal strokes over a ten-year time frame. ${ }^{29}$

$$
<10.0 \% \text { : low }
$$

$10.0-<20.0 \%$ : moderate

$20.0-<30.0 \%$ : high

$30.0-<40.0 \%$ : very high

$\geq 40.0 \%$ : harmfully high

12. Physical activities are defined as: physical movements using body energy. These activities are divided into three levels by intensity. ${ }^{30}$

A high level: vigorous physical activities for $\geq 3$ days/ week, and total metabolic equivalent (MET) minutes per week $\geq 1,500$, or vigorous or moderate physical activities for $\geq 7$ days/week, and MET minutes per week $\geq 3,000$
A moderate level: moderate to high physical activities and vigorous activities for $\geq 3$ days/week, and $\geq 20$ minutes/day, or moderate physical activities, or walking for $\geq 5$ days/week and at least 30 minutes/day, or vigorous or moderate activities or walking for $\geq 5$ days/week, and total MET minutes per week $\geq 600$.

A low level: below the average of moderate and high physical activities.

13. Knowledge scores of stroke were marked via a 20-question questionnaire. The questionnaire was designed to be a 3-choice survey item, which were scored by 1 point for each correct answer and 0 points for incorrect or unsure answers. Based on groups, the scores were separated into $80.0 \%$ for a high level of stroke knowledge, $60.0-79.0 \%$ for a moderate level and less than $60.0 \%$ for a low level. This questionnaire was checked for content validity by three experts, and reliability by the method of Cronbach's Alpha Coefficient with a value of 0.82 .

14. Routine food means some food which is always eaten at least three times per week.

Salty food means some food containing more than one teaspoon in a day.

Sweet food means some food containing more than six teaspoons in a day.

Fatty meat means some read meat mixed with white meat.

The data were analyzed by Stata program, so as to find out the following kinds of statistics.

1. Descriptive statistics: frequency distribution and, percentage.

2. Inferential statistics: the relationships between factors and lipid profiles of other members in stroke patient families, by chi-square test.

3. Descriptions of directions and relationships, by multivariate logistic regression analysis. 


\section{Results}

There were 67 families of stroke patients taking part in this research. The samples were 120 family members, 1-2 members in each family. Details: the samples were 76 blood relatives and 44 non-blood relatives in the patients' families.

\section{Characteristics of the study population}

1. General Information (Table 1 and 2)

There were 120 subjects in total: 31 males and 89 females. There were 54 female and 22 male blood relatives, counted as $45.0 \%$ and $18.3 \%$, respectively. The average age was $48.52 \pm 14.57$ years. The largest number of educational background was primary school level (49.2\%), followed by secondary school level (19.2\%), and high school level (15.0\%). A average incomes of 10,000-15,000 Baht/month made up $40.8 \%$, followed by less than 5,000 Baht/month $(38.3 \%)$. Most $(75.8 \%)$ of the samples had no underlying diseases. Twenty-nine of the samples (24.2\%) had some underlying diseases, divided into 20 samples (16.7\%) with HT; 7 (5.8\%) with DM type 2, one with chronic obstructive pulmonary disease, and one with Epilepsy.

2. Information about health behavior (Table 1)

Overall, the blood relatives had never smoked or consumed alcohol was $50.0 \%$. In terms of annual medical checkups, $38.3 \%$ of the samples had never had any annual medical checkup, followed by $25.8 \%$ of those who had an annual medical checkup, whilst $21.7 \%$ of those went for medical checkup when experiencing some type of symptom, respectively. For the most part, their physical activities were at a low level, $72.5 \%$. Regarding knowledge of stroke, it was found that $54.1 \%$ of the samples had a high level of stroke knowledge, followed by $27.6 \%$ at a moderate level and $18.3 \%$ at a low level, respectively. In addition, the regular food consumed by the subjects were $77.5 \%$ of both vegetables and fish, $65.0 \%$ of fruit, $46.7 \%$ of coffee/ tea, $32.5 \%$ of fatty meats, $23.3 \%$ of high-fat food and food with coconut milk, $18.0 \%$ of salty food, and $11.7 \%$ of sweet food. When the blood relatives and non-blood relatives groups were compared, there were statistically significant differences at a p-value $<0.05$ of age group and educational. Trang Hospital was the most frequent place visited when feeling unwell, followed by subdistrict health centers and private clinics; $31.7 \%, 21.7 \%$, and $20.0 \%$, respectively.

3. Medical checkup information (Table 1 and 2)

The average level of systolic blood pressure was $130.49 \pm 17.62 \mathrm{mmHg}$. Most $(70.0 \%)$ of their systolic blood pressure was <140 mmHg, a normal level; additionally, the average level of diastolic blood pressure was $80.88 \pm$ $12.72 \mathrm{mmHg}$. Their diastolic blood pressure at a normal level was $<90 \mathrm{mmHg}$. The average waist circumstance was $87.21 \pm 11.54 \mathrm{~cm}$. Average BMI was $25.65 \pm 4.22 \mathrm{~kg} / \mathrm{m}^{2}$. Therefore, it was indicated that $27.5 \%$ of the samples had a normal BMI level, $31.7 \%$ of them suffered from Class 1 obesity, and $15.8 \%$ of them faced Class 2 obesity; including risks of cardiovascular disease. According to Thai CV risk scores, the results revealed that $80.8 \%$ of the samples had a low risk, at $<10.0 \%$.

\section{Laboratory Results and preihovalence of} dyslipidemia (Table 3 and 4)

Average, total cholesterol levels were 224.28 \pm 48.20 (Table 2). From the samples, the prevalence of high total cholesterol ( $\geq 240 \mathrm{mg} / \mathrm{dL}$ ) was $36.7 \%$; the prevalence of borderline-high total cholesterol (200-239 mg/dL) was $29.2 \%$; the prevalence of desirable total cholesterol (<200 mg/dL) was $34.2 \%$.

Average HDL-C was 51.64 \pm 12.56 (Table 2). From the samples, the prevalence of low HDL-C (a HDL-C level lower than $40 \mathrm{mg} / \mathrm{dL}$ ) was $15.8 \%$. Average LDL-C was 147.12 \pm 39.06 (Table 2). From the samples, the prevalence of high LDL-C ( $\geq 160 \mathrm{mg} / \mathrm{dL})$ was $37.5 \%$. The prevalence of the optimal LDL-C level was $11.7 \%$, and the prevalence of $100-159 \mathrm{mg} / \mathrm{dL}$ LDL-C was $50.8 \%$. 
Table 1 Characteristics of the study population

\begin{tabular}{|c|c|c|c|}
\hline \multirow[b]{2}{*}{ Variable } & \multicolumn{3}{|c|}{ Family members of stroke patients } \\
\hline & $\begin{array}{l}\text { Blood relatives } \\
(n=76) \\
\text { Number }(\%)\end{array}$ & $\begin{array}{l}\text { Non blood relatives } \\
(n=44) \\
\text { Number }(\%)\end{array}$ & p-value \\
\hline \multicolumn{4}{|l|}{ Sex } \\
\hline Female & $54(45.0)$ & $35(29.2)$ & 0.306 \\
\hline Male & $22(18.3 \%)$ & $9(7.5)$ & \\
\hline \multicolumn{4}{|l|}{ Age group (years) } \\
\hline$<35$ & $21(17.5)$ & $3(2.5)$ & $0.009^{*}$ \\
\hline $35-60$ & $44(36.7)$ & $28(23.3)$ & \\
\hline$>60$ & $11(9.2)$ & $13(10.8)$ & \\
\hline \multicolumn{4}{|l|}{ Education } \\
\hline No education & $2(1.7)$ & $1(0.8)$ & $0.041^{*}$ \\
\hline Primary school level & $30(25.0)$ & $29(24.2)$ & \\
\hline Secondary school level & $14(11.7)$ & $4(3.3)$ & \\
\hline High school level & $17(14.2)$ & $6(5.0)$ & \\
\hline Diploma & $8(6.7)$ & $0(0.0)$ & \\
\hline Bachelor's Degree & $5(4.2)$ & $4(3.3)$ & \\
\hline \multicolumn{4}{|l|}{ Occupational } \\
\hline Employee & $15(12.5)$ & $9(7.5)$ & 0.337 \\
\hline Trading career & $14(11.7)$ & $6(5.0)$ & \\
\hline Farmer & $32(26.7)$ & $13(10.8)$ & \\
\hline Worked at home & $14(11.7)$ & $15(12.5)$ & \\
\hline Civil servant & $1(0.8)$ & $1(0.8)$ & \\
\hline \multicolumn{4}{|l|}{ Income (Baht/year) } \\
\hline$<5,000$ & $26(21.7)$ & $20(16.7)$ & 0.321 \\
\hline $5,000-10,000$ & $13(10.8)$ & $7(5.8)$ & \\
\hline $10,001-15,000$ & $35(29.2)$ & $14(11.7)$ & \\
\hline$>15,000$ & $2(1.7)$ & $3(2.5)$ & \\
\hline \multicolumn{4}{|l|}{ Underlying disease } \\
\hline Yes & $19(15.8)$ & $10(8.3)$ & 0.828 \\
\hline No & $57(47.5)$ & $34(28.3)$ & \\
\hline \multicolumn{4}{|l|}{ Systolic blood pressure $(\mathrm{mmHg})$} \\
\hline$<140$ & $52(43.3)$ & $32(26.7)$ & 0.836 \\
\hline $140-159$ & $21(17.5)$ & $10(8.3)$ & \\
\hline$\geq 160$ & $3(2.5)$ & $2(1.7)$ & \\
\hline \multicolumn{4}{|l|}{ Diastolic blood pressure $(\mathrm{mmHg})$} \\
\hline$<90$ & $57(47.5)$ & $33(27.5)$ & 0.749 \\
\hline $90-99$ & $13(10.8)$ & $6(5.0)$ & \\
\hline$\geq 100$ & $6(5.0)$ & $5(4.2)$ & \\
\hline \multicolumn{4}{|l|}{$\mathrm{BM} \overline{\mathrm{Il}}$} \\
\hline$<18.5 \mathrm{~kg} / \mathrm{m}^{2}:$ underweight & $2(1.7)$ & $1(0.8)$ & 0.805 \\
\hline $18.5-22.9 \mathrm{~kg} / \mathrm{m}^{2}:$ healthy weight & $19(15.8)$ & $14(11.7)$ & \\
\hline $23-24.9 \mathrm{~kg} / \mathrm{m}^{2}$ : overweight & $18(15.0)$ & $9(7.5)$ & \\
\hline $25-29.90 \mathrm{~kg} / \mathrm{m}^{2}$ : Class 1 obesity & $23(19.2)$ & $15(12.5)$ & \\
\hline$\geq 30 \mathrm{~kg} / \mathrm{m}^{2}:$ Class 2 obesity & $14(11.7)$ & $5(4.2)$ & \\
\hline \multicolumn{4}{|l|}{ Random plasma glucose (mg\%) } \\
\hline$<180$ & $74(61.7)$ & $43(35.8)$ & 0.903 \\
\hline$\geq 180$ & $2(1.7)$ & $1(0.8)$ & \\
\hline \multicolumn{4}{|l|}{ Regular food consumed by the subjects } \\
\hline High fat food and food with coconut & $18(15.0)$ & $10(8.3)$ & 0.905 \\
\hline Fatty meats & $29(24.2)$ & $10(8.3)$ & 0.082 \\
\hline Sweet food & $10(8.3)$ & $4(3.3)$ & 0.504 \\
\hline Salty food & $13(10.8)$ & $5(6.6)$ & 0.396 \\
\hline
\end{tabular}


Table 1 (continued)

\begin{tabular}{|c|c|c|c|}
\hline \multirow[b]{2}{*}{ Variable } & \multicolumn{3}{|c|}{ Family members of stroke patients } \\
\hline & $\begin{array}{l}\text { Blood relatives } \\
(n=76)\end{array}$ & $\begin{array}{l}\text { Non blood relatives } \\
(n=44) \\
\text { Number }(\%)\end{array}$ & p-value \\
\hline Coffee/tea & $39(32.5)$ & $17(14.2)$ & 0.180 \\
\hline Dessert & $14(11.7)$ & $9(7.5)$ & 0.785 \\
\hline Fish & $59(49.2)$ & $34(28.3)$ & 0.964 \\
\hline Fruit & $49(40.8)$ & $29(24.2)$ & 0.876 \\
\hline Vegetables & $60(50.0)$ & $33(27.5)$ & 0.618 \\
\hline \multicolumn{4}{|l|}{ Physical activities } \\
\hline A high level & $5(4.2)$ & $2(1.7)$ & 0.734 \\
\hline A moderate level & $15(12.5)$ & $11(9.2)$ & \\
\hline A low level & $56(46.7)$ & $31(25.8)$ & \\
\hline \multicolumn{4}{|l|}{ Current smoking state $(\%)$} \\
\hline Never & $60(50.0)$ & $38(31.7)$ & 0.312 \\
\hline Past or current & $16(13.3)$ & $6(5.0)$ & \\
\hline \multicolumn{4}{|l|}{ Current drinking state (\%) } \\
\hline Never & $60(50.0)$ & $38(31.7)$ & 0.312 \\
\hline Past or current & $16(13.3)$ & $6(5.0)$ & \\
\hline \multicolumn{4}{|l|}{ Annual medical checkup } \\
\hline Never & $31(25.8)$ & $15(12.5)$ & 0.636 \\
\hline Checkup in case of some disorders & $17(14.2)$ & $9(7.5)$ & \\
\hline Once a year & $18(15.0)$ & $13(10.8)$ & \\
\hline 2 times/year & $2(1.7)$ & 0 & \\
\hline$>2$ times/year & $8(6.7)$ & $7(5.8)$ & \\
\hline \multicolumn{4}{|l|}{ Knowledge scores of stroke } \\
\hline High level & $12(10.0)$ & $10(8.3)$ & 0.520 \\
\hline Moderate level & $23(19.2)$ & $10(8.3)$ & \\
\hline Low level & $41(34.2)$ & $24(20.0)$ & \\
\hline \multicolumn{4}{|l|}{ Thai CV risk score $(\%)$} \\
\hline$<10$ & 64 (53.3) & $33(27.5)$ & 0.067 \\
\hline $10-<20$ & $6(5.0)$ & $6(5.0)$ & \\
\hline $20-<30$ & $2(1.7)$ & $5(4.2)$ & \\
\hline$\geq 30$ & $4(3.3)$ & $0(0.0)$ & \\
\hline
\end{tabular}

${ }^{*}$ Chi-square test

Note: Data are expressed as means \pm S.D. or $\mathrm{n}(\%)$.

$\mathrm{BMI}=$ body mass index, TC=total cholesterol, HDL-C=high-density lipoprotein cholesterol, LDL-C=low-density lipoprotein cholesterol, $\mathrm{SBP}=$ systolic blood preesure, $\mathrm{DBP}=$ dystolic blood preesure, $\mathrm{FBG}=$ fasting blood glucose, Thai $\mathrm{CV}$ risk score=Thai cardiovascular risk score

The prevalence of dyslipidemia calculated from at least one type of an unusual level of cholesterol (LDL-C $\geq 160 \mathrm{mg} / \mathrm{dL}$ or $\mathrm{HDL}-\mathrm{C}<40 \mathrm{mg} / \mathrm{dL}$ or total cholesterol $\geq 240$ $\mathrm{mg} / \mathrm{dL}$ ) was $51.7 \%$. In terms of random plasma glucose, average blood sugar was 104.43+36.88 (Table 2). 
Table 2 Mean of the study population

\begin{tabular}{ll}
\hline Variable & Mean \pm S.D. \\
\hline Average age (years) & $48.52 \pm 14.57$ \\
Systolic blood pressure $(\mathrm{mmHg})$ & $130.49 \pm 17.62$ \\
Diastolic blood pressure $(\mathrm{mmHg})$ & $80.88 \pm 12.72$ \\
Waist circumstance $(\mathrm{cm})$ & $87.21 \pm 11.54$ \\
Body mass index $\left(\mathrm{kg} / \mathrm{m}^{2}\right)$ & $25.65 \pm 4.22$ \\
Random blood glucose $(\mathrm{mg} / \mathrm{dL})$ & $104.43 \pm 36.88$ \\
Total cholesterol $(\mathrm{mg} / \mathrm{dL})$ & $224.28 \pm 48.20$ \\
$<240$ & $194.83 \pm 29.76$ \\
$\geq 240$ & $275.14 \pm 26.58$ \\
HDL-C (mg/dL) & $51.64 \pm 12.56$ \\
$<40$ & $35.63 \pm 3.02$ \\
$\geq 40$ & $54.65 \pm 11.32$ \\
LDL-C $(\mathrm{mg} / \mathrm{dL})$ & $147.12 \pm 39.06$ \\
$<160$ & $122.33 \pm 23.66$ \\
$\geq 160$ & $188.42 \pm 19.85$ \\
\hline
\end{tabular}

$\mathrm{mmHg}=$ millimeters of mercury, $\mathrm{cm}=$ centimeter, BMl=body mass index, $\mathrm{kg} / \mathrm{m}^{2}=$ kilograms divided by height in meters squared, $\mathrm{mg} / \mathrm{dL}=$ milligram per deciliter, HDL-C=high-density lipoprotein cholesterol, LDL-C=high density lipoprotein cholesterol

Table 3 Laboratories result of the study population

\begin{tabular}{|c|c|c|c|}
\hline \multirow[b]{2}{*}{ Variable } & \multicolumn{3}{|c|}{$\begin{array}{l}\text { Family members of stroke patients } \\
\qquad(n=120)\end{array}$} \\
\hline & $\begin{array}{l}\begin{array}{l}\text { Blood } \\
\text { relatives } \\
(n=76)\end{array} \\
\text { Number (\%) }\end{array}$ & $\begin{array}{l}\text { Non-blood } \\
\text { relatives } \\
(n=44) \\
\text { Number (\%) }\end{array}$ & $p$-value \\
\hline \multicolumn{4}{|c|}{ Cholesterol (mg/dL) } \\
\hline $\begin{array}{l}<200 \\
200-239 \\
\geq 240\end{array}$ & $\begin{array}{ll}26 & (21.6) \\
23 & (19.2) \\
27 & (22.5)\end{array}$ & $\begin{array}{l}15(12.5) \\
12(10.0) \\
17(14.2)\end{array}$ & 0.733 \\
\hline \multicolumn{4}{|c|}{$\mathrm{HDL}-\mathrm{C}(\mathrm{mg} / \mathrm{dL})$} \\
\hline $\begin{array}{l}<40 \\
\geq 40\end{array}$ & $\begin{array}{l}10(8.3) \\
66(55.0)\end{array}$ & $\begin{array}{l}9(7.5) \\
35(29.2)\end{array}$ & 0.291 \\
\hline \multicolumn{4}{|c|}{ LDL-C (mg/dL) } \\
\hline $\begin{array}{l}<100 \\
100-159 \\
\geq 160\end{array}$ & $\begin{array}{l}8(6.7) \\
40(33.3) \\
28(23.3)\end{array}$ & $\begin{array}{l}6(5.0) \\
21(17.5) \\
17(14.2)\end{array}$ & 0.845 \\
\hline \multicolumn{4}{|l|}{ Dyslipidemia } \\
\hline $\begin{array}{l}\text { Yes } \\
\text { No }\end{array}$ & $\begin{array}{l}35(29.2) \\
41(34.2)\end{array}$ & $\begin{array}{l}27(22.5) \\
17(14.2)\end{array}$ & 0.106 \\
\hline
\end{tabular}

$\mathrm{mg} / \mathrm{dL}=$ milligram per deciliter, $\mathrm{HDL}-\mathrm{C}=$ =high-density lipoprotein cholesterol, LDL-C=high density lipoprotein cholesterol
Table 4 Prevalence of blood cholesterol level

\begin{tabular}{|c|c|c|c|}
\hline \multirow[b]{2}{*}{ Variable } & \multicolumn{3}{|c|}{ Prevalence } \\
\hline & $\begin{array}{l}\text { Blood } \\
\text { relatives } \\
(n=76)\end{array}$ & $\begin{array}{l}\text { Non-blood } \\
\text { relatives } \\
(n=44)\end{array}$ & $\begin{array}{l}\text { Family } \\
\text { members } \\
\text { of stroke } \\
(n=120)\end{array}$ \\
\hline \multicolumn{4}{|c|}{ Total Cholesterol (mg/dL) } \\
\hline$<200$ & $34.2 \%$ & $34.1 \%$ & $34.2 \%$ \\
\hline $200-239$ & $30.3 \%$ & $27.3 \%$ & $29.2 \%$ \\
\hline$\geq 240$ & $35.5 \%$ & $36.6 \%$ & $36.7 \%$ \\
\hline \multicolumn{4}{|c|}{$\mathrm{HDL}-\mathrm{C}(\mathrm{mg} / \mathrm{dL})$} \\
\hline$<40$ & $13.1 \%$ & $20.5 \%$ & $15.8 \%$ \\
\hline$\geq 40$ & $86.8 \%$ & $79.5 \%$ & $84.2 \%$ \\
\hline \multicolumn{4}{|c|}{ LDL-C (mg/dL) } \\
\hline$<100$ & $10.5 \%$ & $13.6 \%$ & $11.7 \%$ \\
\hline $100-159$ & $52.6 \%$ & $47.7 \%$ & $50.8 \%$ \\
\hline$\geq 160$ & $36.8 \%$ & $38.6 . \%$ & $37.5 \%$ \\
\hline \multicolumn{4}{|l|}{ Dyslipidemia } \\
\hline Yes & $46.1 \%$ & $61.4 \%$ & $51.7 \%$ \\
\hline No & $53.9 \%$ & $38.6 \%$ & $48.3 \%$ \\
\hline
\end{tabular}

$\mathrm{mmHg}=$ millimeters of mercury, $\mathrm{cm}=$ centimeter, $\mathrm{BMl}=$ body mass index, $\mathrm{Kg} / \mathrm{m}^{2}=\mathrm{kilograms}$ divided by height in meters squared, $\mathrm{mg} / \mathrm{dL}=$ milligram per deciliter, HDL-C=high-density lipoprotein cholesterol, LDL-C=high density lipoprotein cholesterol

\section{Dyslipidemia and risk factors (Table 5)}

In this study, out of 120 patients, there were 62 patients with dyslipidemia (51.7\%) and 58 patients without dyslipidemia. The participants of both groups liked to consume the same foods: vegetables and fish. The percentage of dyslipidemia people who preferred fish, vegetables, and fruits was $77.5,77.4$, and 61.3 , respectively. These results were not significantly different from the results of people with normal lipid levels, calculated as $77.5 \%, 77.5 \%$, and $69.0 \%$, respectively. Most of the dyslipidemia samples did a few physical activities (74.2\%). Likewise, most of the normal-cholesterol samples performed a few physical activities (70.7\%). Like normalcholesterol participants, most of dyslipidemia participants had never consume alcohol or smoked. In terms of their annual medical checkup, $45.2 \%$ of the dyslipidemia subjects 
Table 5 Dyslipidemia and risk factors

\begin{tabular}{|c|c|c|c|c|}
\hline \multirow[b]{2}{*}{ Characteristic } & \multicolumn{2}{|c|}{ Dyslipidemia $(n=62)$} & \multicolumn{2}{|c|}{ Non-dyslipidemia $(n=58)$} \\
\hline & $\begin{array}{l}\text { Relatives } \\
(n=35) \\
\text { Number (\%) }\end{array}$ & $\begin{array}{l}\text { Not relatives } \\
(n=27) \\
\text { Number }(\%)\end{array}$ & $\begin{array}{l}\text { Relatives } \\
(n=41) \\
\text { Number }(\%)\end{array}$ & $\begin{array}{l}\text { Not relatives } \\
(n=17) \\
\text { Number }(\%)\end{array}$ \\
\hline \multicolumn{5}{|l|}{ The regular foods consumed by the subjects } \\
\hline High-fat food and food with coconut milk & $6(9.7)$ & $7(11.3)$ & $12(20.7)$ & $3(5.2)$ \\
\hline Fatty meat & $11(17.7)$ & $5(8.1)$ & $18(31.0)$ & $5(8.6)$ \\
\hline Sweet foods & $4(6.5)$ & $1(1.6)$ & $6(10.3)$ & $3(5.2)$ \\
\hline Salty foods & $6(9.7)$ & $3(4.8)$ & $7(12.1)$ & $2(3.4)$ \\
\hline Coffee/tea & $17(27.4)$ & $10(16.1)$ & $22(37.9)$ & $7(12.1)$ \\
\hline Dessert & $8(12.9)$ & $4(6.5)$ & $6(10.3)$ & $5(8.6)$ \\
\hline Fish & $28(45.2)$ & $20(32.3)$ & $31(53.4)$ & $14(24.1)$ \\
\hline Vegetables & $29(46.8)$ & $19(30.6)$ & $31(53.4)$ & $14(24.1)$ \\
\hline Fruit & $21(33.9)$ & $17(27.4)$ & $28(48.3)$ & $12(20.7)$ \\
\hline \multicolumn{5}{|l|}{ Physical activities } \\
\hline A high level & $3(4.8)$ & $1(1.6)$ & $2(3.4)$ & $1(1.7)$ \\
\hline A moderate level & $8(12.9)$ & $4(6.5)$ & $7(12.1)$ & $7(12.1)$ \\
\hline A low level & $24(38.7)$ & $22(35.5)$ & $32(55.2)$ & $9(15.5)$ \\
\hline \multicolumn{5}{|l|}{ Tobacco use } \\
\hline Never & $26(41.9)$ & $23(37.1)$ & $34(58.6)$ & $15(25.9)$ \\
\hline Past or current & $9(14.5)$ & $4(6.5)$ & $7(12.1)$ & $2(3.4)$ \\
\hline \multicolumn{5}{|l|}{ Alcohol use } \\
\hline Never & $25(40.3)$ & $23(37.1)$ & $35(60.3)$ & $15(25.9)$ \\
\hline Past or current & $10(16.1)$ & $4(6.5)$ & $6(10.3)$ & $2(3.4)$ \\
\hline \multicolumn{5}{|l|}{ annual medical checkup } \\
\hline Never & $15(24.2)$ & $13(21.0)$ & $16(27.6)$ & $2(3.4)$ \\
\hline Checkup in case of some disorders & $10(16.1)$ & $4(6.5)$ & $7(12.1)$ & $5(8.6)$ \\
\hline Once a year & $5(8.1)$ & $7(11.3)$ & $13(22.4)$ & $6(10.3)$ \\
\hline 2 times/year & $0(0.0)$ & $0(0.0)$ & $2(3.4)$ & $0(0.0)$ \\
\hline$>2$ times/year & $5(8.1)$ & $3(4.8)$ & $3(5.2)$ & $4(6.9)$ \\
\hline \multicolumn{5}{|l|}{ Knowledge scores of stroke } \\
\hline High level & $8(12.9)$ & $5(8.1)$ & $4(6.9)$ & $5(8.6)$ \\
\hline Moderate level & $9(14.5)$ & $6(9.7)$ & $14(24.1)$ & $4(6.9)$ \\
\hline Low level & $18(29.0)$ & $16(25.8)$ & $23(39.7)$ & $8(9.1)$ \\
\hline \multicolumn{5}{|l|}{$\mathrm{BMI}$} \\
\hline <18.5: underweight & $0(0.0)$ & $0(0.0)$ & $2(3.4)$ & $1(1.7)$ \\
\hline 18.5-22.9: healthy weight & $11(17.7)$ & $10(16.1)$ & $8(13.8)$ & $4(6.9)$ \\
\hline 23-24.9: overweight & $8(12.9)$ & $6(9.7)$ & $10(17.2)$ & $3(5.2)$ \\
\hline 25-29.90: Class 1 obesity & $11(17.7)$ & $8(12.9)$ & $12(20.7)$ & $7(12.1)$ \\
\hline$\geq 30:$ Class 2 obesity & $5(8.1)$ & $3(4.8)$ & $9(15.5)$ & $2(3.4)$ \\
\hline \multicolumn{5}{|l|}{ Thai Cardiovascular risk score } \\
\hline$<10 \%$ : low & $28(45.2)$ & $19(30.6)$ & $36(62.1)$ & $14(24.1)$ \\
\hline 10-<20\%: moderate & $4(6.5)$ & $5(8.1)$ & $2(3.4)$ & $1(1.7)$ \\
\hline $20-<30 \%$ : high & $1(1.6)$ & $3(4.8)$ & $1(1.7)$ & $2(3.4)$ \\
\hline $30-<40 \%$ : very high & $2(3.2)$ & $0(0.0)$ & $2(3.4)$ & $0(0.0)$ \\
\hline$>40 \%$ : harmfully high & $0(0.0)$ & $0(0.0)$ & $0(0.0)$ & $0(0.0)$ \\
\hline
\end{tabular}

BMI=body mass index 
had never gone for an annual medical checkup, followed by $22.6 \%$ of those who went for checkups in case of some disorder and $19.4 \%$ of those who went once a year. In the group of normal-cholesterol subjects, $32.8 \%$ of them had a checkup once a year, $31.0 \%$ of them had never done any checkup, and $20.7 \%$ of them went for a checkup if there were some symptoms of illness. In regards to knowledge of stoke and cardiovascular disease, there were no statistically significant differences between both groups. According to the Thai CV risk scores, the participants in both groups had a lot of knowledge as well as, less possibility of cardiovascular disease. Regarding BMI, most of the subjects, in both groups, had a healthy weight. Only $22.6 \%$ of dyslipidemia subjects risked Class 1 obesity, while $30.7 \%$ risked Class 2 obesity. In normal-cholesterol subjects $22.4 \%$ were overweight, $30.7 \%$ had Class 1 obesity, and
$13.0 \%$ had Class 2 obesity. Interestingly, there were more dyslipidemia subjects maintaining a healthy weight than the normal-cholesterol subjects, $33.9 \%$ and $20.7 \%$, respectively. There was the highest number of normal-cholesterol subjects having Class 1 obesity while there was the largest number of dyslipidemia subjects being of a healthy weight.

\section{Factors related to dyslipidemia (Table 6)}

When the factors related to the dyslipidemia people (cholesterol $\geq 240 \mathrm{mg} / \mathrm{dL}$ or HDL-C $<40 \mathrm{mg} / \mathrm{dL}$ or LDL-C $\geq 160 \mathrm{mg} / \mathrm{dL}$ ) were determined by the multivariate model, it was found that the factors were underlying diseases. The patients with some underlying diseases were more likely to have dyslipidemia than those patients without underlying disease (adjusted odd ratio=6.13, 95\% Cl=2.27-16.55).

Table 6 Factors related to dyslipidemia

\begin{tabular}{|c|c|c|c|}
\hline \multirow{2}{*}{ Variables } & \multicolumn{3}{|c|}{ Dyslipidemia } \\
\hline & Odds ratio & p-value & $95 \% \mathrm{Cl}$ \\
\hline \multicolumn{4}{|l|}{ Gender } \\
\hline Male & 1.00 & & \\
\hline Female & 0.45 & 0.592 & $0.03-8.12$ \\
\hline \multicolumn{4}{|l|}{ Age (years) } \\
\hline$<35$ & 1.00 & & \\
\hline $30-60$ & 0.99 & 0.994 & $0.43-2.26$ \\
\hline$>60$ & 1.44 & 0.441 & $0.56-3.69$ \\
\hline \multicolumn{4}{|l|}{ Current smoking state } \\
\hline No & 1.00 & & \\
\hline Yes & 0.61 & 0.79 & $0.01-25.07$ \\
\hline \multicolumn{4}{|l|}{ Current drinking state } \\
\hline No & 1.00 & & \\
\hline Yes & 2.79 & 0.497 & $0.14-54.04$ \\
\hline \multicolumn{4}{|l|}{ Underlying disease } \\
\hline No & 1.00 & & \\
\hline Yes & 6.13 & $0.000^{*}$ & $2.27-16.55$ \\
\hline \multicolumn{4}{|l|}{ Educational level } \\
\hline No education/Primary school & 1.00 & & \\
\hline High school/Bachelor's degree & 0.87 & 0.724 & $0.42-1.79$ \\
\hline \multicolumn{4}{|l|}{$\mathrm{BMI}$} \\
\hline Normal BMI & 1.00 & & \\
\hline Abnormal BMI & 0.68 & 0.340 & $0.30-1.49$ \\
\hline
\end{tabular}


Table 6 (continued)

\begin{tabular}{|c|c|c|c|}
\hline \multirow{2}{*}{ Variables } & \multicolumn{3}{|c|}{ Dyslipidemia } \\
\hline & Odds ratio & $p$-value & $95 \% \mathrm{Cl}$ \\
\hline \multicolumn{4}{|l|}{ Waist circumstance $(\mathrm{cm})$} \\
\hline$<90$ (male)/80 (female) & 1.00 & & \\
\hline$\geq 90($ male)/80 (female) & 8.05 & 0.064 & $0.89-73.17$ \\
\hline \multicolumn{4}{|l|}{ Systolic blood pressure $(\mathrm{mmHg})$} \\
\hline$<140$ & 1.00 & & \\
\hline $140-159$ & 1.15 & 0.728 & $0.50-2.64$ \\
\hline$\geq 160$ & 0.63 & 0.629 & $0.10-4.00$ \\
\hline \multicolumn{4}{|l|}{ Diastolic blood pressure $(\mathrm{mmHg})$} \\
\hline$<90$ & 1.00 & & \\
\hline $90-99$ & 0.94 & 0.904 & $0.35-2.53$ \\
\hline$\geq 100$ & 4.70 & 0.056 & $0.96-22.99$ \\
\hline \multicolumn{4}{|l|}{ Physical activities } \\
\hline A high level & 1.00 & & \\
\hline A moderate level & 0.20 & 0.319 & $0.00-4.72$ \\
\hline A low level & 0.26 & 0.702 & $0.03-11.15$ \\
\hline \multicolumn{4}{|l|}{ Relatives suffered from stroke } \\
\hline No & 1.00 & & \\
\hline Yes & 2.37 & 0.537 & $0.25-1.14$ \\
\hline \multicolumn{4}{|l|}{ Annual medical checkup } \\
\hline Never/checkup in case of some disorders & 1.00 & & \\
\hline Once a year $/ \geq 2$ times/year & 0.73 & 0.607 & $0.22-2.37$ \\
\hline \multicolumn{4}{|l|}{ Knowledge scores of stroke } \\
\hline High level & 1.00 & & \\
\hline Moderate level & 0.51 & 0.501 & $0.07-3.58$ \\
\hline Low level & 1.26 & 0.776 & $0.25-6.28$ \\
\hline \multicolumn{4}{|l|}{ Thai cardiovascular risk score (\%) } \\
\hline \multicolumn{4}{|l|}{$<10$} \\
\hline$\geq 10$ & 1.06 & 0.952 & $0.14-7.86$ \\
\hline \multicolumn{4}{|l|}{ Regular food consumed by the subjects } \\
\hline High-fat food and food with coconut milk & 0.33 & 0.272 & $0.05-2.34$ \\
\hline Fatty meats & 0.53 & 0.486 & $0.09-3.18$ \\
\hline Sweet food & 0.70 & 0.774 & $0.06-7.88$ \\
\hline Salty food & 0.85 & 0.869 & $0.12-5.97$ \\
\hline Coffee/tea & 0.85 & 0.815 & $0.22-3.33$ \\
\hline Dessert & 1.21 & 0.825 & $0.23-6.42$ \\
\hline Fish & 0.47 & 0.503 & $0.05-4.33$ \\
\hline Fruit & 0.34 & 0.235 & $0.06-2.01$ \\
\hline Vegetables & 2.94 & 0.312 & $0.36-23.72$ \\
\hline
\end{tabular}

$\mathrm{Cl}=$ confidence interval, $\mathrm{mmHg}=$ millimeters of mercury, $\mathrm{BMl}=$ body mass index 


\section{Discussion}

Currently, there have been few of studies on cholesterol levels of family members of stroke patients. The studies of dyslipidemia in Thailand were different according to patients' age ranges and characteristics. For example, a study in 2000 revealed that $21.1 \%$ of working adults had dyslipidemia. ${ }^{31}$ In contrast, when male elderly people were diagnosed, the prevalence of total cholesterol at >200 $\mathrm{mg} / \mathrm{dL}$ and $\mathrm{LDL} \geq 160 \mathrm{mg} / \mathrm{dL}$ was $87.5 \%$ and $55.4 \%$, respectively. For female elderly people, the prevalence of total cholesterol at $\geq 200 \mathrm{mg} / \mathrm{dL}$ and $\mathrm{LDL} \geq 160 \mathrm{mg} / \mathrm{dL}$ was $100.0 \%$ and $80 \%$, respectively. ${ }^{32}$ Therefore, the aforementioned results were different from this study results due to the smaller number of working adults of study as above. In fact, epidemiology of dyslipidemia in Thailand was high total cholesterol, high LDL-C, Low HDL-C and high triglyceride. $^{33}$ In this study, although high total cholesterol and high LDL-C were similarly found, only $15.8 \%$ of low HDL-C was found. HDL-C at $\geq 40 \mathrm{mg} / \mathrm{dL}$ was $84.2 \%$ in this research. Regarding the study by Suchat et al. ${ }^{34}$ during 2004-2006 (Thai Epidemiologic study), it was found that the prevalence of hyperlipidemia (fasting cholesterol $\geq 200 \mathrm{mg} / \mathrm{dL}$ ) in stroke patients and non-stroke patients was $77.1 \%$ and $65.7 \%$, respectively. The results of the non-stroke patients were similar to the samples of this research-65.8\% of patients with hyperlipidemia (cholesterol $\geq 200 \mathrm{mg} / \mathrm{dL}$ ).

According to Choowong et al. $(2005)^{35}$, it was claimed that prevalence of hypercholesterolemia ( $\geq 200 \mathrm{mg} / \mathrm{dL}$ ), low HDL-C (<40 mg/dL), and high LDL-C ( $\geq 130 \mathrm{mg} / \mathrm{dL})$ was found in $31.0 \%, 14.0 \%$, and $20.0 \%$ of citizens who lived in rural areas of Khon Kaen. The prevalence of low $\mathrm{HDL}$ in the abovementioned research and in this research was similar. On the other hand, the prevalence of hypercholesterolemia and high LDL found in this study was higher. Referring to the study by Wichai et al. $(2009)^{33}$, 19,021 samples over-20-year-old patients, were looked at, and the results showed that the prevalence of high LDL-C and low HDL-C was $29.6 \%$ and $47.1 \%$, respectively. Differently, the prevalence of high $L D L-C$ in this study was higher (37.5\%) and the prevalence of low HDL-C was lower (15.8\%). In terms of dyslipidemia, this study results were related to the study by Pornpimon et al. $(2017)^{36}$. The samples were 44 patients at the age of 3560 who lived in Khon Kaen. The prevalence of LDL-C at $\geq 130 \mathrm{mg} / \mathrm{dL}$ was $41.2 \%$, but it was higher in this study-75 patients with dyslipidemia $(62.5 \%)$ compared with the previous studies showed that trends in prevalence of lipid abnormality was increased likely due to national advancement, convenient transportation, and information access, which may contrite and be consequences of dietary changes. ${ }^{31-39}$ Nevertheless, all the mentioned comparisons were inconclusive since the number of samples in each research were not alike. It was suggested that there should be more samples in this research. The oversea study of the prevalence of dyslipidemia among stroke patients by lyad et al. $(2015)^{40}$ in Palestine revealed that $28.6 \%$ of patients had high LDL-C ( $\geq 130 \mathrm{mg} / \mathrm{dL}), 17.1 \%$ had high total cholesterol $(\geq 200 \mathrm{mg} / \mathrm{dL})$, and $61.3 \%$ had low HDL $(<40$ $\mathrm{mg} / \mathrm{dL}$ ). It could be seen in this study when compared to lyad et al. (2015) that the prevalence of high cholesterol and high LDL-C was higher and the prevalence of low HDL-C was lower. Regarding the prevalence of LDL-C, even though the prevalence of high LDL-C was not high $(37.5 \%)$, there were $106(88.3 \%)$ out of 120 patients who had higher than optimal LDL-C ( $\geq 100 \mathrm{mg} / \mathrm{dL})$.

This finding is alarming that build the awareness of this risk group is necessary because the highest number of subjects with dyslipidemia were those that had never undergone a medical checkup, thus appropriate screening, checkup and therapeutic lifestyle change programs for high-risk groups must be scaled up. The factor related to dyslipidemia was underlying diseases. Hypertension (16.7\%) was the highest proportion in this 
study, followed by diabetes mellitus (5.8\%). The chronic diseases were likely to affect dyslipidemia.

In terms of stroke knowledge, there were no statistically significant differences between blood relatives and non-blood relative samples, nor between dyslipidemia and non-dyslipidemia samples. The participants in every group mostly knew a lot of stroke information; nevertheless, having a lot of stroke knowledge could not predict that they would not have dyslipidemia. Concerning risks of cardiovascular disease in the next ten years, via the Thai CV risk score, although the samples of this study had low physical activities and high BMI which was considered as obesity, the assessment results indicated that most of the samples were ranked as low risk possibly due to younger ages (adults), in that they are at a lower risk level than their elderly counrerparts. According to the data of the National Health Survey in the Thai population, the prevalence of hypercholesterolemia increased with age from below 10.0\% in young adults, to about $25.0 \%$ in the elderly. ${ }^{32}$ In addition, most of the samples in this study did not smoke, and their waist circumference and systolic blood pressure were not above the criteria; hence, the CV risks were low. Prevalence of blood relatives and non-blood relatives of stroke patient were no difference; they are the same family so regular food preferences, there were no statistically significant differences between blood relatives and non-blood relatives subjects, with a similar percentage having the same dietary habits. Even though the samples had knowledge in regards to health problems related to high-fat food; including food with coconut milk, fatty meats, and tea/coffee, dyslipidemia was still a factor for them. However, there was a high percentage of fish, vegetable, and fruit eaten, and a low percentage of salty/sweet food consumed in these two groups. Therefore, there was high prevalence of dyslipidemia in the family members of stroke patients, including conditions of overweight and prehypertension. The causes of dyslipidemia could be genetic conditions, and consumption behaviors of each family. This could have an influence on health behaviors. In consequence, care for stroke patients should not only include medical treatments, but primary prevention, secondary prevention coupled with, health promotion should also be provided to patients' relatives and family members.

\section{Conclusion}

Most family members of stroke patients in this study were of a working age; they had no underlying diseases, and were blood relatives of the stroke patients, but had a low level of physical activities. Most samples did not drink alcohol or smoke, additionally most did not prefer to have regular medical checkups, or just preferred to visit a medical center only when they were feeling unwell. Most of them had a high level of stroke knowledge, apart from diets containing high-fat food, fatty meats, food with coconut milk, and tea/coffee. Also most liked to eat fish, vegetables and fruit. Furthermore, most of them were overweight, and had prehypertension. Prevalence of dyslipidemia in family Members of Stroke Patients was $51.7 \%$. Detection of this abnormality is important to them. This would help in designing a prevention plan, as well as to raise awareness of stroke prevention in family members of stroke patients.

\section{Conflict of interest}

There were no potential conflicts of interest to declare.

\section{References}

1. Papol A, Boonkaew N. Comparison of stroke risk assessment tools. EAU Heritage J 2018;12:62-70.

2. Saicamthon P, Tipsriraj S, Chaisaen K, Yingyong T, Sugawong K. The effect of risk factors for cerebrovascular disease, regional health 1, Thailand. Lanna Public Health J 2016;12: 44-5.

3. Lindsay P, Furie KL, Davis SM, Donnan GA, Norrving B. World stroke organization global stroke services guidelines and action plan. J Stroke 2014;9:4-13. 
4. Watkins LO. Epidemiology and burden of cardiovascular disease. Clin Cardiol 2004;27(Suppl 3):III2-6.

5. Gordon DJ, Probstfield JL, Garrison RJ, Neaton JD, Castelli WP. High-density lipoprotein cholesterol cardiovascular disease. Four prospective American studies. Circulation 1989; 79:8-15.

6. Verschuren WM, Boerma GJ, Kromhout D. Total and HDLcholesterol in The Netherlands: 1987-1992. Levels and changes over time in relation to age, gender and educational level. Int J Epidemiol 1994;23:948-56.

7. Assmann G, Schulte H, von Eckardstein A, Huang Y. Highdensity lipoprotein cholesterol as a predictor of coronary heart disease risk. The PROCAM experience and pathophysiological implications for reverse cholesterol transport. Atherosclerosis 1996;124(Suppl):11-20.

8. Goldbourt U, Yaari S, Medalie JH. Isolated low HDL cholesterol as a risk factor for coronary heart disease mortality. A 21-year follow-up of 8000 men. ATVB 1997;17:107-13.

9. Eastern Stroke Coronary Heart Disease Collaborative Research Group. Blood pressure, cholesterol, and stroke in eastern Asia. Lancet 1998;352:1801-7.

10. Stamler J, Daviglus ML, Garside DB, Dyer AR, Greenland P. Relationship of baseline serum cholesterol levels in 3 large cohorts of younger men to long-term coronary, cardiovascular, and all-cause mortality and to longevity. JAMA 2000;284: 311-8.

11. Expert Panel on Detection, Evaluation, and Treatment of High Blood Cholesterol in Adults. Executive summary of the third report of the national cholesterol education program (NCEP) expert panel on detection, evaluation, and treatment of high blood cholesterol in adults (adult treatment panel III). JAMA 2001;285:2486-97.

12. Bhuripanyo K, Tatsanavivat $\mathrm{P}$, Matrakool B, Muktabhant B, Bhuripanyo P, Harnthaveesompol S. A prevalence survey of lipids abnormalities of rural area in Amphoe Phon, Khon Kaen. J Med Assoc Thai 1993;76:101-8.

13. Lindenstrom E, Boysen G, Nyboe J. Influence of total cholesterol, high density lipoprotein cholesterol, and triglycerides on risk of cerebrovascular disease: the Copenhagen City Heart Study. BMJ 1994;309:11-5.

14. Chaisiri K, Pongpaew P, Tungtrongchitr R, Phonrat B, Kulleap $S$, Sutthiwong $P$, et al. Nutritional status and serum lipids of a rural population in Northeast Thailand-an example of health transition. Int J Vitam Nutr Res 1998:68:196-202.

15. Stamler J, Daviglus ML, Garside DB, Dyer AR, Greenland P, Neaton JD. Relationship of baseline serum cholesterol levels in 3 large cohorts of younger men to long-term coronary, cardiovascular, and all-cause mortality and to longevity. JAMA 2000;284:311-8.

16. Yamwong $\mathrm{P}$, Assantachai $\mathrm{P}$, Amornrat A. Prevalence of dyslipidemia in the elderly in rural areas of Thailand. Southeast Asian J Trop Med Public Health 2000;31:158-62

17. Yusuf $S$, Reddy $S$, Ounpuu $S$, Anand S. Global burden of cardiovascular diseases: part I: general considerations, the epidemiologic transition, risk factors, and impact of urbanization. Circulation 2001;104:2746-53.

18. Pongchaiyakul $\mathrm{C}$, Pongchaiyakul $\mathrm{C}$, Pratipanawatr T. Prevalence of dyslipidemia in rural Thai adults: an epidemiologic study in Khon Kaen province. J Med Assoc Thai 2005;88:1092-7.

19. Marshall J. Familial incidence of cerebral hemorrhage. Stroke 1973;4:38-41.

20. Khaw Kay-T, Barrett-Connor E. Family history of stroke as an independent predictor of ischemic heart disease in men and stroke in women. Am J Epidemiol 1986;123:59-66.

21. Howard G, Evans G, Toole JF, Tell J, Rose L, Espeland M, et al. Characteristics of stroke victims associated with early cardiovascular mortality in their children. J Clin Epidemiol 1990;43:49-54

22. Brass LM, Shaker LA. Family history in patients with transient ischemic attacks. Stroke 1991;22:837-41.

23. Graffagnino C, Gasecki AP, Doig GS, Hachinski VC. The importance of family history in cerebrovascular disease. Stroke 1994;25:1599-604.

24. World Health Organization. The Asia-Pacific perspective redefining obesity and its treatment [monograph on the Internet]. Geneva: WHO; 2000 [cited 2019 Jun 11]. Available from: https://apps.who.int//ris/bitstream/handle/10665/ 206936/0957708211_eng.pdf? sequence=1\&isAllowed=y

25. Roche Diagnostics GmbH. Cholesterol CHOD-PAP method. Mannheim: Roche Diagnostics GmbH; 2000.

26. Roche Diagnostics $\mathrm{GmbH}$. HDL-Cholesterol and LDLCholesterol plus. Mannheim: Roche Diagnostics GmbH; 2000.

27. Michael SL, Phil BF. Expert Panel on Detection, Evaluation, and Treatment of High Blood Cholesterol in Adults. Executive Summary of the Third Report of the National cholesterol Education Program (NCEP) Expert Panel on Detection, Evaluation, and 
Treatment of High Blood Cholesterol in Adults (Adult Treatment Panel III). JAMA 2001;285:2486-97.

28. O’Brien E, Petrie J, Littler W, de Swiet M, Padfield PL, O'Malley $\mathrm{K}$, et al. The British Hypertension Society protocol for the evaluation of automated and semi-automated blood pressure measuring devices with special reference to ambulatory systems. J Hypertens 1990;8:607-19.

29. Chobanian AV, Bakris GL, Black HR, Cushman WC, Green LA, Izzo JL, et al. The seventh report of the joint nationa committee on prevention, detection, evaluation, and treatment of high blood pressure: the JNC 7 Report. JAMA 2003;289: 2560-72.

30. World Health Organization. Physical activity [homepage on the Internet]. Geneva: WHO; 2015 [cited 2018 Feb 23]. Available from: https://www.who.int/en/news-room/fact-sheets/detail/ physical-activity

31. Bhuripanyo K, mahanonda N, Leowattana W, Ruangratanaamporn O, Sriratanasathavorn C, Chotinaiwattarakul C, et al. A 5-year prospective study of conventional risk factors of coronary artery disease in Shinawatra employees: a preliminary prevalence survey of 3,615 employees. J Med Assoc Thai 2000;83(Suppl 2):S98-105.

32. Yamwong P, Assantachai P, Amornrat A. Prevalence of dyslipidemia in the elderly in rural areas of Thailand. Southeast Asian J Trop Med Public Health 2000;31:158-62.

33. Wichai A, Surasak T, Pattapong K, Virasakdi C, Panwadee P, Piyamitr $\mathrm{S}$, et al. Prevalence of dyslipidemia and management in the Thai Population, National Health Examination Survey IV, 2009 [monograph on the Internet]. London: Hindawi; 2014 [cited 2020 Jun 11]. Available from: http://downloads.hindawi. com/journals/Jl/2014/249584.pdf
34. Suchat H, Niphon P, Samart N, Nijasri S, Pimchanok P, Somchai $\mathrm{T}$, et al. Prevalence of stroke and stroke risk factors in Thailand: Thai Epidemiologic Stroke (TES) study. J Med Assoc Thai 2011; 94:427-36.

35. Choowong P, Chatlert P, Thongchai P. Prevalence of dyslipidemia in rural Thai adults: an epidemiologic study in Khon Kaen Province. J Med Assoc Thai 2005;88:1089-9.

36. Pornpimon C, Benja M, Frank PS. Prevalence and factors associated with non-high density lipoprotein cholesterol among Thai adults in Khon Kaen Province of Northeastern Thailand. J Public Health Development 2017;15:49-61.

37. Bhuripanyo K, Tatsanavivat P, Matrakool B, Muktabhant B, Bhuripanyo P, Harnthaveesompol S. A prevalence survey of lipids abnormalities of rural area in Amphoe Phon, Khon Kaen. J Med Assoc Thai 1993;76:101-8.

38. Chaisiri K, Pongpaew P, Tungtrongchitr R, Phonrat B, Kulleap S, Sutthiwong $P$, et al. Nutritional status and serum lipids of a rural population in Northeast Thailand-an example of health transition. Int J Vitam Nutr Res 1998;68:196-202.

39. Pongchaiyakul C, Hongsprabhas P, Pisprasert V. Rural-urban difference in lipid levels and prevalence of dyslipidemia: a population-based study in Khon Kaen province, Thailand. J Med Assoc Thai 2006;89:1835-44.

40. Iyad A, Mahmoud A, Anan A, Omar S, Ahmad AS, Sabreen M, et al. The prevalence of dyslipidemia and hyperglycemia among stroke patients: preliminary findings [homepage on the Internet]. London: Hindawi; 2015 [cited 2020 Jan 20]. Available from: https://www.ncbi.nlm.nih.gov/pmc/articles/PMC6875342/ pdf/SRT2019-8194960.pdf 